\title{
Malaria chemoprophylaxis in travellers to east Africa: a comparative prospective study of chloroquine plus proguanil with chloroquine plus sulfadoxine-pyrimethamine
}

\author{
S FOGH， A SCHAPIRA， I C BYGBJERG，S JEPSEN， C H MORDHORST， K KUIJLEN, \\ P RAVN, A RØNN， P C GØTZSCHE
}

\begin{abstract}
As malaria caused by Plasmodium falciparum has become resistant to chloroquine alternative drug regimens need to be developed. The prophylactic efficacy against malaria and the side effects of chloroquine phosphate $500 \mathrm{mg}$ weekly with proguanil hydrochloride $200 \mathrm{mg}$ daily was compared with the efficacy of chloroquine $500 \mathrm{mg}$ weekly with sulfadoxine $\mathbf{5 0 0}$ mg-pyrimethamine $25 \mathrm{mg}$ weekly in a randomised study of Scandinavian travellers to Kenya and Tanzania during 1984-5. A total of 767 subjects (416 male and 351 female; 384 taking chloroquine phosphate with proguanil hydrochloride and 383 taking chloroquine with sulfadoxine-pyrimethamine) completed a diary on the breakthrough of malaria and the side effects of treatment while taking the drugs. They were also asked to make thick blood films when symptoms like those of malaria occurred, which were sent to and analysed in Denmark. Four subjects taking chloroquine with proguanil hydrochloride and three taking chloroquine with sulfadoxine-pyrimethamine developed falciparum malaria, which was verified microscopically. Side effects were reported by 36 subjects taking chloroquine phosphate with proguanil hydrochloride and 55 taking the other regimen $(p=0 \cdot 043)$. The side effects of both regimens were generally mild, but the combination of chloroquine phosphate with proguanil hydrochloride is recommended because it results in fewer side effects.

As breakthroughs of malaria occurred at the earliest after seven weeks self treatment should not be recommended for travellers staying only a short time. Thick blood films are useful for diagnosis of suspected cases of malaria, can be prepared by non-specialists in Africa, and can be analysed successfully after long delays.
\end{abstract}

\section{Introduction}

Malaria due to Plasmodium falciparum resistant to chloroquine was first reported in non-immune visitors to east Africa in $1979 .{ }^{1}$ As a consequence the Danish national recommendation for chemo-

WHO National Malaria Diagnostic Centre, Malaria Research Laboratory, Department of Clinical Microbiology, and Vaccination Centre for Travellers, Statens Seruminstitut, Copenhagen, Denmark

$S$ FOGH, MD, DTM\&H, senior registrar

A SCHAPIRA, MD, DTM\&H, research fellow

S JEPSEN, MD, DTM\&H, senior research fellow

C H MORDHORST, MD, head, vaccination centre for travellers

Department of Communicable and Tropical Diseases, Rigshospitalet, Copenhagen

IC BYGBJERG, MD, DTM\&H, senior registrar

P RAVN, MB

A RØNN, MB

PC GØTZSCHE, MD, MSC, registrar

Nordic Health Clinic, Dar es Salaam, Tanzania

K KUIJLEN, MD, DTM\&H, physician

Correspondence to: Dr S Jepsen, WHO National Malaria Diagnostic Centre, Statens Seruminstitut, 2300-S Copenhagen, Denmark. prophylaxis against malaria was changed from chloroquine $300 \mathrm{mg}$ base weekly to chloroquine $600 \mathrm{mg}$ base weekly. The annual national statistics for the next four years, however, showed a steady increase in the number of cases of imported falciparum malaria, especially from east Africa. After the report in 1982 on the resistance of falciparum malaria to sulfadoxine-pyrimethamine (Fansidar) the recommendations for malaria chemoprophylaxis were changed to chloroquine $300 \mathrm{mg}$ base weekly and sulfadoxine-pyrimethamine one tablet weekly. ${ }^{2}$ During the next year a reduction by more than half in the number of imported cases of falciparum malaria was observed, although the number of Danish travellers remained unchanged. Because of the reports of possible serious side effects when sulfadoxine-pyrimethamine is used as prophylaxis, ${ }^{3}$ and as the efficacy and possible side effects of the combinations of chloroquine with proguanil and chloroquine with sulfadoxine-pyrimethamine have never been studied, we undertook a randomised trial of the two drug regimens.

\section{Subjects and methods}

From May 1984 to September 1985 travellers to and temporary residents in Tanzania or Kenya, or both, who presented at the vaccination centres in Denmark and the Nordic Health Clinic, Dar es Salaam, were asked to participate in a randomised study of two prophylactic antimalarial drug regimens-namely, chloroquine phosphate $500 \mathrm{mg}$ (two tablets) weekly with proguanil hydrochloride $200 \mathrm{mg}$ (two tablets daily; subjects weighing more than $90 \mathrm{~kg}$ received $300 \mathrm{mg}$ (three tablets)) versus chloroquine (two tablets) with sulfadoxine $500 \mathrm{mg}$-pyrimethamine $25 \mathrm{mg}$ (one tablet), both taken weekly. The drugs were not masked. The doses given to children varied with age. Before entering the trial each participant was given thorough oral and written information according to the Helsinki II declaration. The protocol was approved by the Danish Ethics Committee.

Exclusion criteria included allergy to sulphonamides, pregnancy, infancy (those aged less than 4 weeks), and unwillingness to take either regimen. Those who agreed to take part in the trial were randomly assigned to one of the prophylactic regimens according to a table of random numbers. In the case of families all members were given the same regimen. Prophylaxis was to be taken from one week before entering countries where malaria was endemic to four weeks after leaving them. Each person was given a diary and asked to fill in the route of travel, duration of stay in malarious areas, any side effects of the prophylaxis, and any feverish episodes. The travellers were also supplied with a "malaria kit." This consisted of a strong padded envelope, which contained a plastic tube with three tablets of mefloquine hydrochloride $250 \mathrm{mg}$ with sulfadoxine $500 \mathrm{mg}$-pyrimethamine $25 \mathrm{mg}$ (Fansimef), a plastic box with three microscope slides and sterile lancets, and written instructions on how to make two thick blood films (or how to let another person do so) should symptoms similar to those of malaria occur. The subjects were asked to take the drug in the tube after making a blood film, which was sent by post to Denmark. When possible local physicians in Nairobi, Mombasa, Dar es Salaam, and Zanzibar were asked to confirm the diagnosis of malaria before treatment and report the findings in the diary.

In Denmark the prophylactic drugs were prescribed and bought at pharmacies. In Dar es Salaam they were supplied free of charge at the clinic to those who entered the study.

Assuming a breakthrough of malaria of $1 \%$ in the group taking chloroquine with sulfadoxine-pyrimethamine $\left(\mathrm{p}_{1}=0.01\right)$ and a minimally relevant difference of $3 \%\left(p_{2}=0.04\right)$, then with a type I error risk of $5 \%$ and a type II error risk of $10 \%$ the required number of participants in each group was 633 (normal approximation to the binomial with continuity correction). ${ }^{4}$

Duration of stay in east Africa was compared with the Gaussian approximation to the Mann-Whitney test. Two by two tables were analysed with Fisher's exact test, and confidence intervals for proportions were 
calculated with the Gaussian approximation; for calculations of malaria breakthrough we used the Poisson distribution as well as the Gaussian approximation.

\section{Results}

A total of 1009 subjects ( 555 male and 454 female) agreed to participate. Of these, 242 were subsequently excluded (129 from the group taking chloroquine with sulfadoxine-pyrimethamine and 113 from that taking chloroquine phosphate with proguanil hydrochloride) for the following reasons: travel cancelled, 19; diary not returned, 170; diary not completed properly (that is, information lacking on duration of travel or drug treatment or on symptoms ascribed to malaria or drug treatment), 38; and other reasons (that is, unwillingness to participate in spite of initial acceptance because of advice to stop the drug treatment or to take alternative drugs from local residents or doctors, or both), 15 . More than $80 \%$ of the participants were recruited at the vaccination centre at Statens Seruminstitut, Copenhagen, and about $10 \%$ at the Nordic Health Centre, Dar es Salaam.

The study comprised 767 subjects (416 male and 351 female), 384 of whom were given chloroquine phosphate with proguanil hydrochloride and 383 chloroquine with sulfadoxine-pyrimethamine; 476 went to Kenya, 188 to Tanzania, and 103 to both countries (table I). The median age in both groups was 35 years $(95 \%$ range, $5-65)$. The duration of stay in east Africa was similar in the two groups $\left(p=0.36, z^{\prime}=0.91\right)$. About $70 \%$ of the subjects in both groups paid short visits (less than four weeks), and about $5 \%$ were recruited among long term residents in Tanzania.

TABLE I-Countries visited by subjects receiving prophylaxis against malaria

\begin{tabular}{lcc}
\hline & $\begin{array}{c}\text { Group taking chloroquine } \\
\text { phosphate with proguanil } \\
\text { hydrochloride }(\mathrm{n}=384)\end{array}$ & $\begin{array}{c}\text { Group taking chloroquine } \\
\text { with sulfadoxine-pyrimethamine } \\
(\mathrm{n}=383)\end{array}$ \\
\hline Kenya & 240 & 236 \\
Tanzania & 94 & 94 \\
Kenya and Tanzania & 50 & 53 \\
\hline
\end{tabular}

In the group taking chloroquine phosphate with proguanil hydrochloride four subjects developed falciparum malaria, which was verified microscopically; one did so after returning to Denmark. This last man may have received insufficient doses of the drugs as he took only two proguanil hydrochloride tablets despite weighing $97 \mathrm{~kg}$. Three subjects receiving chloroquine with sulfadoxine-pyrimethamine developed verified falciparum malaria (table II); one of them had stopped taking sulfadoxinepyrimethamine on her return to Denmark, although she had continued to take chloroquine. Thus the total incidence of breakthrough of malaria was

TABLE II-Details of subjects receiving prophylaxis against malaria who subsequently developed malaria

\begin{tabular}{cccc}
\hline Sex & Age (years) & Countries visited & $\begin{array}{c}\text { Breakthrough } \\
\text { time (weeks) }\end{array}$ \\
\hline \multicolumn{4}{c}{ Chloroquine phosphate with proguanil hydrochloride } \\
F & 47 & Kenya and Tanzania & 33 \\
$M$ & 56 & Tanzania & 33 \\
$M$ & 60 & Kenya and Tanzania & 7 \\
Tanzania & 23 \\
F & 37 & Tanzan & \\
F & Chloroquine with sulfadoxine-pyrimethamine & 8 \\
M & 27 & Kenya and Tanzania & 11 \\
& 24 & Kenya and Tanzania & 24 \\
\hline
\end{tabular}

$0.9 \%$; the approximated $95 \%$ confidence interval for the difference between the drug regimens was $-1 \%$ to $1 \cdot 5 \%$ which was well within the minimally relevant difference of $3 \%$ stipulated before the trial. Alternatively, as the approximation was poor owing to low numbers and assuming that the true incidence of breakthrough with chloroquine with sulfadoxinepyrimethamine was $1 \%$ as estimated before the trial, then the $95 \%$ confidence interval for breakthrough of malaria with chloroquine phosphate with proguanil hydrochloride was $0 \cdot 3 \%$ to $2 \cdot 7 \%$.

Eight subjects in the group taking chloroquine phosphate with proguanil hydrochloride had suspected malaria; six were found not to be infected when the slides sent to Denmark were examined, and slides were not taken in the two others. The corresponding figure in the group taking chloroquine with sulfadoxine-pyrimethamine was seven subjects, of whom three had negative blood films and the four others had not made blood films.

Side effects were reported by $36(9 \%)$ subjects in the group taking chloroquine phosphate with proguanil hydrochloride and by $55(14 \%)$ in the group taking chloroquine with sulfadoxine-pyrimethamine $(p=0.043$, $95 \%$ confidence interval for the difference $0.4 \%$ to $9 \cdot 6 \%$ ). Side effects were reported by almost twice as many female as male subjects. Fifty side effects were listed by the group taking chloroquine phosphate with proguanil hydrochloride and 84 by the other group (table III). Serious side effects were

TABLE III-Number and type of side effects experienced by subjects during prophylaxis against malaria

\begin{tabular}{|c|c|c|c|}
\hline Side effects & $\begin{array}{c}\text { Group taking chloroquine } \\
\text { phosphate with proguanil } \\
\text { hydrochloride }\end{array}$ & $\begin{array}{l}\text { Group taking chloroquine } \\
\text { with sulfadoxine- } \\
\text { pyrimethamine }\end{array}$ & Total \\
\hline Skin & 3 & 8 & 11 \\
\hline Eruption & 3 & 6 & 9 \\
\hline Itching & & 1 & 1 \\
\hline Flare up of psoriasis & & 1 & 1 \\
\hline Gastrointestinal & 23 & 27 & 50 \\
\hline Nausea & 13 & 13 & 26 \\
\hline Vomiting & 3 & 5 & 8 \\
\hline Diarrhoea & 7 & 8 & 15 \\
\hline Constipation & & 1 & 1 \\
\hline Nervous system & 8 & 26 & 34 \\
\hline Disturbed vision & 1 & 6 & 7 \\
\hline Headache & 2 & 7 & 9 \\
\hline Dizziness & 5 & 10 & 15 \\
\hline Labile psyche & & 1 & 1 \\
\hline Nightmares & & 2 & 2 \\
\hline Other & 16 & 23 & 39 \\
\hline Fever & 3 & 5 & 8 \\
\hline Tiredness & 1 & 8 & 9 \\
\hline \multicolumn{4}{|l|}{ Palpitations or } \\
\hline trembling & & 2 & 2 \\
\hline Oedema & & 1 & 1 \\
\hline General discomfort & & 1 & 1 \\
\hline \multirow{2}{*}{\multicolumn{4}{|c|}{ Decreased resistance }} \\
\hline & & 1 & 1 \\
\hline Dysuria & 2 & & 2 \\
\hline Rhinitis & & 1 & 1 \\
\hline Oral ulceration & 1 & & 1 \\
\hline Inability to tan as usual & 2 & & 2 \\
\hline Loss of hair & & 1 & 1 \\
\hline Dry throat & 4 & & 4 \\
\hline Coated tongue & & 1 & 1 \\
\hline Cough & 3 & 1 & 4 \\
\hline Dyspnoea & & $i$ & 1 \\
\hline Total & 50 & 84 & 134 \\
\hline No of subjects affected & 36 & 55 & 91 \\
\hline
\end{tabular}

not observed. Eight subjects stopped taking daily proguanil hydrochloride because of its side effects, and two other subjects stopped taking chloroquine phosphate, although they still continued to take proguanil hydrochloride. Nineteen subjects stopped taking sulfadoxine-pyrimethamine because of its side effects, and another stopped taking chloroquine while continuing to take sulfadoxine-pyrimethamine.

In only four cases were the side effects specifically ascribed to chloroquine; in the remainder no attempt was made to distinguish between side effects caused by the combination of drugs and those caused by each individual drug. Most side effects developed early in both groups (within four weeks). Clustering of side effects was common, also in both groups. In 10 families 23 members complained of similar side effects, predominantly non-specific

TABLE IV-Reasons given for not adhering to prescribed drug regimen. Values are numbers of subjects

\begin{tabular}{lccc}
\hline Reasons & $\begin{array}{c}\text { Group taking chloroquine } \\
\text { phosphate with proguanil } \\
\text { hydrochloride }\end{array}$ & $\begin{array}{c}\text { Group taking chloroquine } \\
\text { with sulfadoxine- } \\
\text { pyrimethamine }\end{array}$ & Total \\
\hline Side effects & 10 & 20 & 30 \\
Forgetfulness & 13 & 15 & 28 \\
Rumour or advice & 7 & 11 & 18 \\
Inconvenience & 2 & 2 & 4 \\
\hline Total & 32 & 48 & 80 \\
\hline
\end{tabular}


gastrointestinal symptoms. Thus about a quarter of all the subjects complaining of side effects belonged to only 10 families.

Altogether 80 subjects did not adhere to the prescribed drug regimen: 32 were in the group receiving chloroquine phosphate and proguanil hydrochloride and 48 in the other. Table IV lists the reasons given for nonadherence to the regimens. Apart from side effects, which were more common in the group receiving chloroquine with sulfadoxine-pyrimethamine $(p=0 \cdot 090,95 \%$ confidence interval for the difference $5 \cdot 4 \%$ to $-0 \cdot 1 \%$ ) there were no obvious differences between the two groups. Several subjects in the group taking chloroquine phosphate with proguanil hydrochloride were advised to change to taking chlorproguanil hydrochloride with or without chloroquine phosphate, and in the other group rumours of side effects and advice to change to taking chlorproguanil hydrochloride or proguanil hydrochloride with or without chloroquine were the main reasons for noncompliance with the drug regimen. In a few cases participants were advised by local residents to take no prophylaxis at all.

\section{Discussion}

Our study shows that there was no obvious difference between the two drug regimens with respect to their protection against malaria. As expected about $1 \%$ of subjects taking chloroquine with sulfadoxine-pyrimethamine developed verified falciparum malaria. The number of participants was less than planned mainly because of difficulties in recruiting subjects in Africa who were not already adapted to one of the regimens or to other regimens-for example, chlorproguanil with or without chloroquine. Besides, we could see that the random recruitment of further participants might be hampered by reports of side effects of chloroquine with sulfadoxinepyrimethamine. ${ }^{3}$ The study, however, had sufficient power to detect a minimal relevant difference between the two regimens.

Falciparum malaria resistant to chloroquine in subjects taking proguanil hydrochloride $200 \mathrm{mg}$ daily has been reported from Vietnam, ${ }^{5}$ and an unacceptably high level of breakthrough with the combination of proguanil hydrochloride and chloroquine has recently been reported from Papua New Guinea: four out of 120 subjects developed falciparum malaria while in Papua New Guinea and one within a few days after returning to Hong Kong, where an additional 14 subjects developed malaria after stopping taking chloroquine and continuing with proguanil hydrochloride alone. ${ }^{6}$

Falciparum malaria resistant to chloroquine and sulfadoxinepyrimethamine has been described previously in east Africa. ${ }^{1278}$ The prevalence of resistance to proguanil hydrochloride is uncertain at present, ${ }^{9}$ but $200 \mathrm{mg}$ daily of this drug was reported to be effective in a retrospective study among non-immune expatriates resident in Dar es Salaam. ${ }^{10}$ Concomitant in vitro resistance of $P$ falciparum to cycloguanil (the active metabolite of proguanil hydrochloride) and pyrimethamine has been reported, ${ }^{11}$ but a relative lack of cross resistance in vitro between cycloguanil and pyrimethamine has also been reported. ${ }^{12}$ The conflicting data may be ascribed to the different culture media used; a medium with low folate and non para-aminobenzoic acid is needed to avoid spurious cross resistance..$^{73}$

Our study was not done blind for practical reasons (proguanil hydrochloride had to be taken daily and sulfadoxine-pyrimethamine weekly, and proguanil hydrochloride has an easily recognisable bitter taste), and several reports of severe side effects of sulfadoxinepyrimethamine early in $1985^{3}$ - that is, in the middle of our studymay have biased the reporting of side effects: a significantly higher number of side effects was reported in the group taking chloroquine with sulfadoxine-pyrimethamine, but, fortunately, no serious side effects occurred. Lifethreatening side effects, even death, have occurred in about one in 20000 people taking sulfadoxinepyrimethamine. ${ }^{14}$ Therefore this drug in combination with chloroquine is no longer recommended by the World Health Organisation for prophylaxis against malaria, ${ }^{14}$ and less toxic alternative regimens are needed. The combination of chloroquine with proguanil hydrochloride is considered to be devoid of serious side effects, ${ }^{9}$ as suggested by this study.

The very small proportion of subjects who forgot to follow the regimens was probably due to the fact that the subjects were motivated for the trial and not merely taking routine prophylaxis.
The combination of a drug to be taken daily (proguanil hydrochloride) with another to be taken weekly (chloroquine phosphate) is hardly ideal. The number of subjects who did not adhere to this regimen because of inconvenience or forgetting to do so, however, was similar to the number of those taking the weekly regimen of chloroquine with sulfadoxine-pyrimethamine. Besides, Watkins et al showed recently that an analogue of proguanil hydrochloride, chlorproguanil hydrochloride, which is given weekly, was ineffective in preventing malaria in east Africa, whether given alone or in combination with chloroquine. ${ }^{15}$

A combination of pyrimethamine-dapsone (Maloprim) with chloroquine has been recommended in other areas with widespread falciparum malaria resistant to chloroquine, but resistance to pyrimethamine-dapsone has been reported from Kenya ${ }^{16}$ and pyrimethamine-dapsone, like sulfadoxine-pyrimethamine, may have serious side effects. ${ }^{17}$

Interestingly, when slides were sent to Denmark they arrived safely, even after long delays, and the thick films were of surprisingly good quality. This system of blood films could possibly be used among non-immune residents in selected areas as a means of identifying the advancing front of chemoresistant falciparum malaria.

In conclusion, this study shows that the combinations of chloroquine phosphate with proguanil hydrochloride and chloroquine with sulfadoxine-pyrimethamine are equally effective as prophylaxis against malaria in Kenya and Tanzania. Although serious side effects were not encountered with either regimen, the combination of chloroquine phosphate with proguanil hydrochloride is recommended because of its less common side effects. We emphasise that the verified cases of malaria developed after seven weeks at the earliest. It is therefore questionable whether self treatment should be recommended for travellers staying a short time.

We thank the staff of the vaccination and health centres in Denmark and east Africa who made this study possible, in particular the association of vaccinators at Statens Seruminstitut, Copenhagen, and the staff at the Nordic Health Clinic, Dar es Salaam. We acknowledge ICI Pharmaceuticals, Great Britain, for supplying proguanil hydrochloride and Hoffman-La Roche, Switzerland, for supplying sulfadoxine-pyrimethamine and mefloquine hydrochloride with sulfadoxine-pyrimethamine. We thank

$\mathrm{Mr} M$ Weis Bentzon for useful suggestions and critical revision of the manuscript and Dr J Anderson for linguistic help.

\section{References}

1 Fogh S, Jepsen S, Effersøe P. Chloroquine-resistant Plasmodium falciparum malaria in Kenya Trans $R$ Soc Trop Med Hyg 1979;73:228-9.

2 Stahel E, Degrémont A, Lagler U. Pyrimethamine/sulfadoxine resistant falciparum malaria acquired at Dar es Salaam, Tanzania. Lancet 1982;i:1118-9.

3 Centers for Disease Control. Revised recommendations for preventing malaria in travellers to areas with chloroquine-resistant Plasmodium falciparum. MMWR 1985;34:185-90.

4 Sachs L. Angewandte Statistik. Berlin: Springer, 1974.

5 Black RH. Malaria in the Australian army in South Vietnam. Med J Aust 1973;i:1265-70.

6 Henderson A, Simon JW, Melia W. Failure of malaria chemoprophylaxis with a proguanilchloroquine combination in Papua New Guinea. Trans R Soc Trop Med Hyg 1986;80:838-40.

7 Spencer HC, Watkins WW, Sixsmith DG, Koech DK, Chulay JD. A new in vitro test for pyrimethamine/sulfadoxine susceptibility of Plasmodium falciparum and its correlation with in pyrimethamine/sulfadoxine susceptibility of Plasmodium
vivo resistance in Kenya. Bull WHO 1984;62:615-21.

8 Schapira A, Bygbjerg IC, Jepsen S, Flachs H, Bentzon MW. The susceptibility of Plasmodium falciparum to sulfadoxine and pyrimethamine: correlation of in vivo and in vitro results. Am $\mathcal{F}$ Trop Med Hyg 1986;35:239-45.

9 Peto TEA, Gilks CF. Strategies for the prevention of malaria in travellers: comparison of dru regimens by means of risk-benefit analysis. Lancet 1986;i:1256-61.

10 McLarty DG, Webber RH, Jaatinen M, et al. Chemoprophylaxis of malaria in non-immun residents in Dar es Salaam, Tanzania. Lancet 1984;ii:656-9.

11 Schapira A. Concomitant resistance to pyrimethamine and cycloguanil of chloroquine-resistant falciparum malaria from east Africa: an in vitro study of 12 isolates. Trans $R$ Soc Trop Med Hyg 1984;78:359-62.

12 Milhous WK, Weatherly NF, Bowdre JH, Desjardins RE. In vitro activities of and mechanisms of resistance to antifol antimalarial drugs. Antimicrob Agents Chemother 1985;27:525-30.

13 Spencer HC, Sixsmith DG, Watkins WW, Koech DK, Chulay JD. In vitro response of Kenyan Plasmodium falciparum to chloroquine in different media. Trans $R$ Soc Trop Med Hyg 1985;79:116-8.

14 World Health Organisation. Vaccination certificate requirements and health advice for international travel. Geneva: WHO, 1986:48.

15 Watkins WM, Brandling-Bennett AD, Oloo AJ, Howells RE, Gilles HM, Koech DK. Inadequacy of chlorproguanil $20 \mathrm{mg}$ per week as chemoprophylaxis for falciparum malaria in Kenya. Lancet 1987; i: $125-8$.

16 Herzog C, Lambert HP, Maudgal D, Warhurst DC, Rogers HJ. Pyrimethamine/dapsone resistant falciparum malaria imported from Kenya Lancet 1982:i:1119-20.

17 Bruce-Chwatt LJ, Hutchinson DB. Maloprim and agranulocytosis. Lancet 1983;ii:1487-8.

(Accepted 20 November 1987 ) 